\title{
Graphene Foam Chemical Sensor System Based on Principal Component Analysis and Backpropagation Neural Network
}

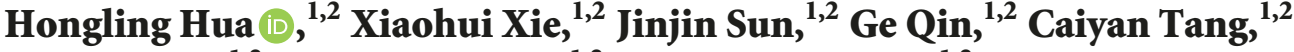 \\ Zhen Zhang, ${ }^{1,2}$ Zhaoqiang Ding, ${ }^{1,2}$ and Weiwei Yue $\mathbb{B}^{1,2}$ \\ ${ }^{1}$ Shandong Province Key Laboratory of Medical Physics and Image Processing Technology, School of Physics and Electronics, \\ Shandong Normal University, Jinan 250014, China \\ ${ }^{2}$ Institute of Materials and Clean Energy, Shandong Normal University, Jinan 250014, China
}

Correspondence should be addressed to Weiwei Yue; yuewei@sdnu.edu.cn

Received 8 December 2017; Accepted 31 January 2018; Published 4 March 2018

Academic Editor: Jiandi Zhang

Copyright (C) 2018 Hongling Hua et al. This is an open access article distributed under the Creative Commons Attribution License, which permits unrestricted use, distribution, and reproduction in any medium, provided the original work is properly cited.

\begin{abstract}
A kind of graphene foam chemical sensor (GFCS) system based on the principal component analysis (PCA) and backpropagation neural network (BPNN) was presented in this paper. Compared with conventional chemical sensors, the GFCS could discriminate various chemical molecules with selectivity without surface modification. The GFCS system consisted of an unmodified graphene foam chemical sensor, an electrical resistance time domain detection system (ERTDS), and a pattern recognition module. The GFCS has been validated via several chemical molecules discrimination including chloroform, acetone, and ether. The experimental results showed that the discrimination accuracy for each molecule exceeded $97 \%$ and a single measurement can be achieved in ten minutes. This work may have presented a new strategy for research and application for graphene chemical sensors.
\end{abstract}

\section{Introduction}

Graphene was a kind of two-dimensional material which has been extensively investigated due to its outstanding performance in terms of high surface-to-volume ratio and outstanding electrical, optical, and mechanical properties [14]. A number of reports were focused on chemical sensing systems based on graphene materials such as chemiresistors $[5,6]$, field-effect transistors $[7,8]$, and other chemical sensors $[9,10]$. Our group also had reported some related works including graphene preparation [11], graphene FET [12], graphene $\mathrm{pH}$ sensors [13], and graphene SPR sensors [14].

Selectivity was one of the fundamental characteristics of chemical sensors. In traditional graphene chemical sensors, it was a common method for improving the selectivity of sensors using the method of surface modification by means of doping with atoms or modification with metal nanoparticles, chemical groups, enzymes/biomolecules, or polymers [1518]. However, the method of surface modification not only increased the complexity of sensor fabrication, but also increased the cost of sensor fabrication. Therefore, graphene chemical sensor without surface modification was a new valuable research direction [19-21]. Rumyantsev et al. reported a graphene transistor using low-frequency noise spectra as an additional sensing parameter to enhance the selectivity of a gas sensor [19]. Nallon et al. fabricated a chemical vapor sensor using the principal component analysis (PCA) to improve the selectivity towards various molecules [20]. Without surface modification, Dobrokhotov et al. constructed a vapor chemiresistor processing the response data of chemiresistor with the Fast Fourier Transform and Quadratic Discriminant Analysis for recognition of acetone, ethanol, and toluene [21]. Furthermore, there were a few theoretical studies reported simulating the interaction between molecules and graphene, which found diverse molecules had various effects on the electrical properties of graphene [22-24]. Those theoretical studies could have offered theoretical supports for studying the selectivity of unmodified graphene.

Due to its porous carbon network structure, threedimensional (3D) graphene foam was an ideal morphology for increasing the active surface area of graphene $[25,26]$. 


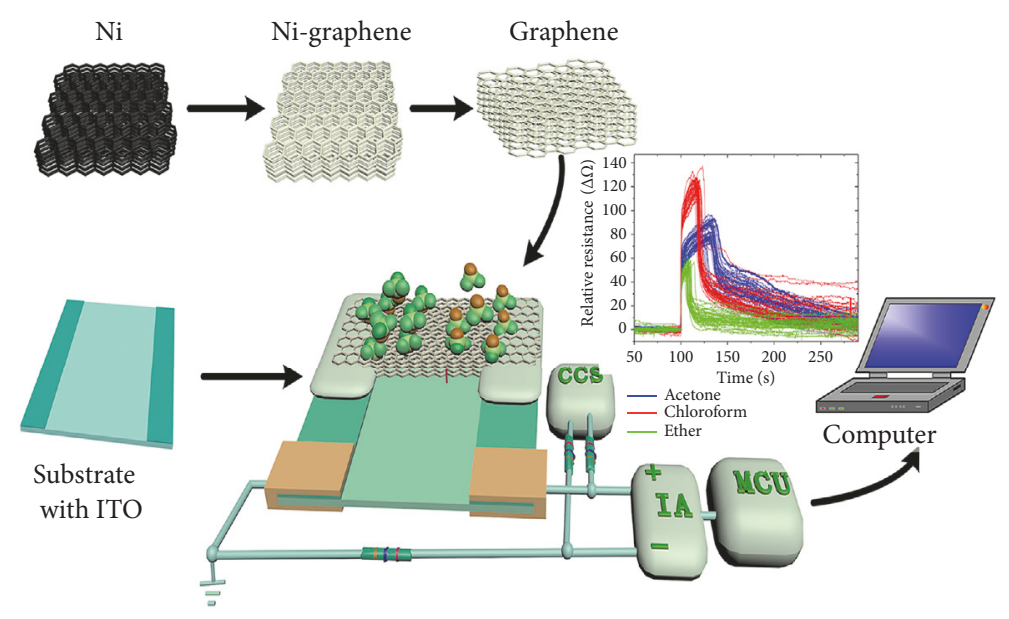

FIGURE 1: The fabrication process of 3D graphene foam and electrical resistance time domain detection system (CCS: constant current source; IA: instrument amplifier; MCU: micro control unit).

In the previous work [27], our group had designed an unmodified graphene foam chemical sensor system based support vector machine (SVM) for making discrimination of different chemical molecules. However, in that work, the extracted characteristics were as input characteristics of SVM directly but did not reduce the complexity of data by further data operation. Thus, to further reduce the complexity of data and improve the efficiency of data processing, the principal component analysis (PCA) $[28,29]$ and the backpropagation neural network (BPNN) [30-32] were used for the graphene foam chemical sensor system (GFCS) in this paper. The PCA was used to reduce the dimension of extracted characteristic, yet the BPNN was used to make pattern recognition for each response curve.

During this work, the unmodified graphene foam was used as sensing element to react with target organic molecules. When the organic molecules of target were introduced to the surface of graphene foam, the resistance measurement system immediately started to record the situation of fluctuant resistance of graphene foam in real time. Then, according to the difference of the time domain resistance curve for various molecules, the GFCS was endued with selectivity after PCA and BPNN.

In this paper, the broad selectivity of GFCS to diverse chemical molecules including acetone, chloroform, and ether had been validated. Furthermore, the fabrication of GFCS, the System of Electrical Resistance Detection, and the algorithm targets discrimination had been described in detail in this paper.

\section{Experimental}

\subsection{Materials and Apparatus}

2.1.1. Materials. The graphene foam with the size of $50 \mathrm{~mm} *$ $50 \mathrm{~mm}$ was purchased from 6-Carbon Technology (Shenzhen, China). Glass substrates modified with indium tin oxide (ITO) were used as substrates for GFCS and they were purchased from Hua Nan Xiang Cheng (Shenzhen,
China) [27]. The silver conductive paint was purchased from CAIG Laboratories (CW-200, USA). Chloroform, ether, and acetone were all of analytical grade and used without further purification.

2.1.2. Apparatus. The 3D graphene foam characterization was carried out by confocal Raman microscopy (SPEX1403, SPEX) [27]. The morphologies of the 3D graphene were characterized by scanning electron microscopy (SEM, FEI Nova Nano450). A homemade electrical resistance time domain detection system (ERTDS) was used to record the graphene resistance curve in real time. All the experiments were carried out at room temperature.

2.2. Fabrication of GFCS. The GFCS was designed based on 3D graphene foam as shown in Figure 1 [27]. The graphene foam was prepared by chemical vapor deposition (CVD) on nickel foam substrate [33-35]. Briefly, graphene films were precipitated on the surface of nickel foam by CVD method. The nickel skeleton was etched away by $\mathrm{FeCl}_{3}$ after deposition of a thin layer of poly (methyl methacrylate) (PMMA) on the surface of graphene films used as supports. The PMMA support was then removed by hot acetone to obtain $3 \mathrm{D}$ graphene foam.

The process of making sensitive components is as follows [27]: Glass substrates modified with ITO were cleaned by ultrasonic agitation in deionized water for 5 minutes. The size of the employed glass substrate was $20 \mathrm{~mm} * 10 \mathrm{~mm}$ and each ITO electrode was $20 \mathrm{~mm} * 2 \mathrm{~mm}$. A piece of graphene foam with the size of $10 \mathrm{~mm} * 5 \mathrm{~mm}$ was attached on the ITO electrodes via silver conductive paint. After drying for 12 hours at room temperature, the silver conductive paint was solidified and the GFCS was ready for measurements.

2.3. Electrical Resistance Time Domain Detection System. Due to diverse adsorption and desorption abilities of different molecules on the interface of graphene, various molecules would produce different shape of time domain dynamic electrical resistance curve on the surface of graphene [27]. Thus, 


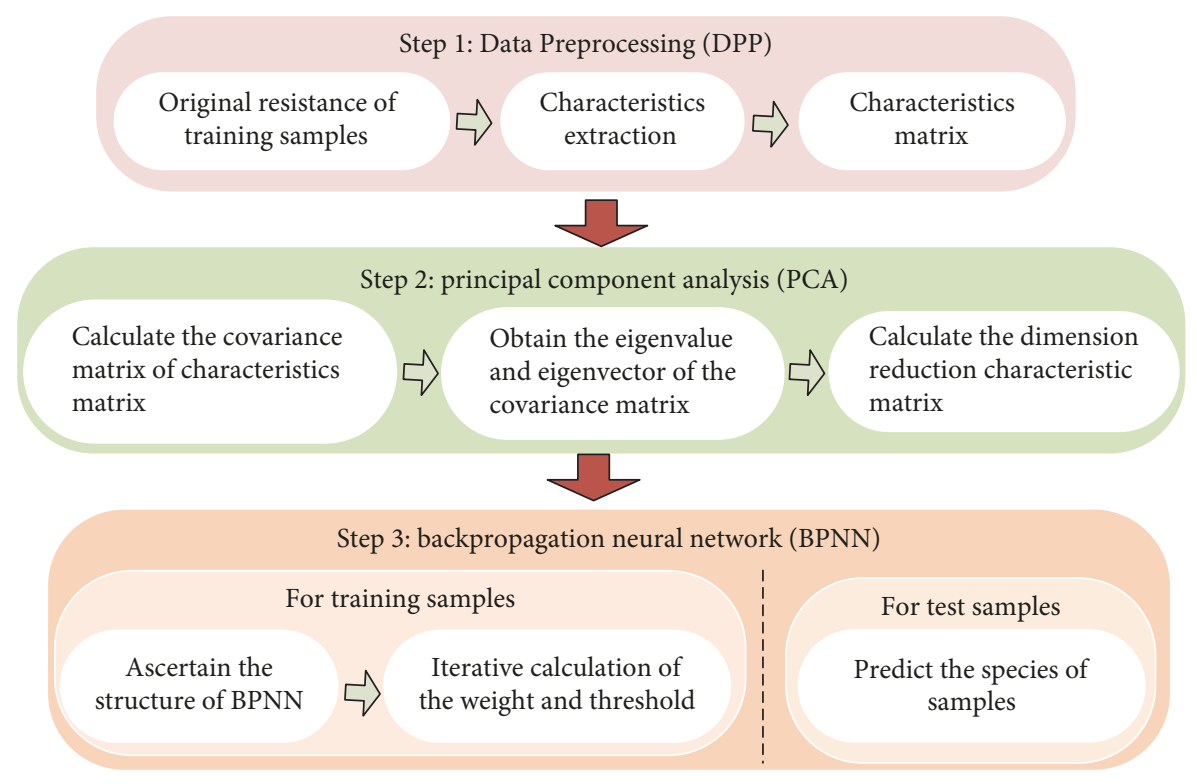

FIGURE 2: The process diagram of the discrimination algorithm.

the ERTDS was constructed to detect the changes of electrical resistance of the GFCS in real time as shown in Figure 1. A Wheatstone bridge circuit and an instrument amplifier were utilized to amplify the tiny fluctuation of resistance $\Delta R$ of GFCS to yield a voltage signal. Next, the amplified voltage signal was converted to digital signal collected by an analog-to-digital (A/D) conversion module ADS1256 and acquired by a micro control unit STM32F103VBT6. For each experiment, the intrinsic resistance of the GFCS was recorded firstly as baseline. Once a kind of chemical molecules was introduced onto the GFCS surface, the real-time resistance curve of GFCS was recorded by the ERTDS and uploaded to the computer via a USB interface.

2.4. Targets Discrimination Algorithm. Real-time resistance curve was used for targets discrimination based on pattern recognition. The algorithm for chemical targets discrimination was composed of Data Preprocessing (DPP), principal component analysis (PCA), and backpropagation neural network (BPNN) as shown in Figure 2.

2.4.1. Data Preprocessing (DPP). DPP was utilized to obtain the matrix characteristics from the resistance curve of GFCS in order to decrease the complexity of original data and improve the efficiency of data processing. A typical time domain dynamic electrical resistance curve of a kind of molecule was shown in Figure 3 [27]. It can be seen clearly from Figure 3 that before introducing the molecules to the graphene foam, graphene resistance remained nearly constant. And following this the molecules were introduced to the surface of graphene foam and the graphene resistance rapidly increased (ascending process). Then after a certain time, the graphene resistance reached a maximum value and

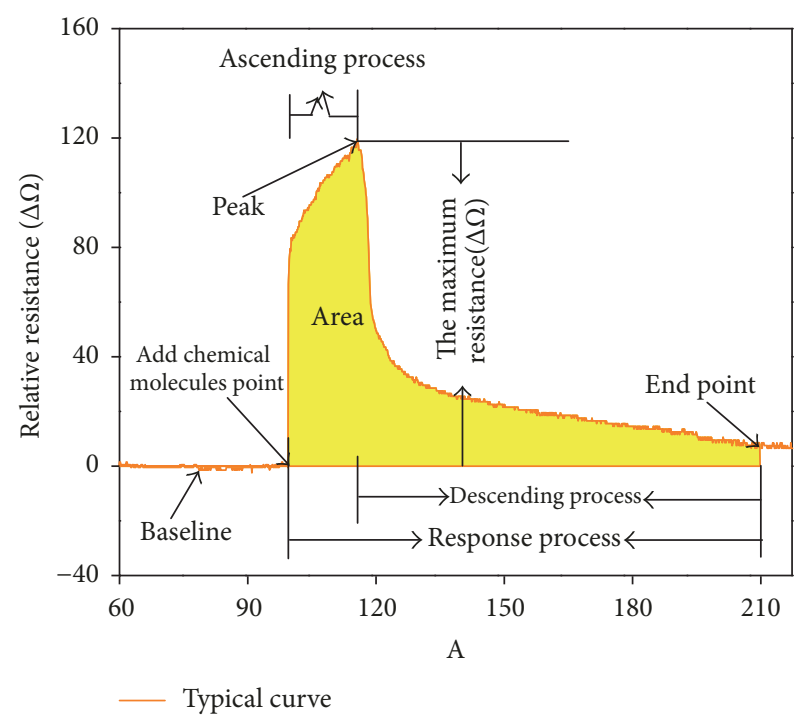

FIGURE 3: The GFCS typical response resistance curve.

followed rapid attenuation (descending process). Finally, the graphene resistance was stabilized to constant value.

For each response curve, ten characteristics could be extracted: time of ascending process $t_{s}$, time of descending process $t_{d}$, time of response process $t$, area of ascending process $A_{s}$, area of descending process $A_{d}$, area of response process $A$, average resistance of ascending process $R_{a s}$, average resistance of descending process $R_{a d}$, biggest change rate of ascending process $V_{s}$, and biggest change rate of descending process $V_{d}$.

Then through the idea of dimension reduction based on PCA, the correlation of ten characteristics would be 


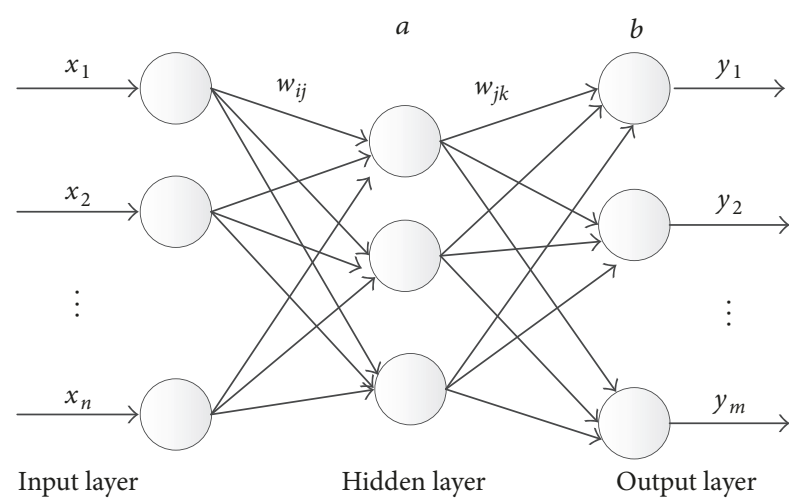

FIGURE 4: The topological structure of BPNN.

further reduced so that it can improve the efficiency of data processing.

2.4.2. Principal Component Analysis. The principal component analysis (PCA), also known as main component analysis, used the idea of dimension reduction to transform multiple related data into a few uncorrelated ones.

In this work, the steps of PCA were as follows: firstly, the covariance matrix of the characteristic matrix which consisted of ten characteristics should be calculated out. Then through the covariance matrix, the eigenvalue and eigenvectors of the characteristic matrix would be obtained. Following this, the vector matrix, which consisted of eigenvectors corresponding to several larger eigenvalues, was obtained. Eventually, the dimension reduction characteristic matrix was calculated out by the product of characteristic matrix and vector matrix.

For training samples, the dimension reduction characteristic matrix was used as the input of BPNNT for obtaining the weights and thresholds of backpropagation neural network (BPNN). For test samples, the dimension reduction characteristics matrix was used as the input of BPNNC for making a classification.

2.4.3. Backpropagation Neural Network. The topological structure of BPNN which consisted of input layer, hidden layer, and output layer was shown in Figure $4 . x_{1}, x_{2}, \ldots, x_{n}$ express the input value of BPNN and each $x_{i}$ was a specific characteristic value, and $y_{1}, y_{2}, \ldots, y_{m}$ represent the prediction values of BPNN. $w_{i j}$ was the weight between the input and hidden layers, while $w_{j k}$ was the weight between hidden and output layers. $a$ and $b$ represent the threshold of hidden layer and output layer, respectively.

The calculation process of BPNN was as follows: firstly, the parameter of the topological structure of BPNN should be determined according to the quantity of the input characteristics and the species of molecules. Then, the weights $w_{i j}$, $w_{j k}$ and the thresholds $a_{j}, b_{k}$ were given randomly. The third step was calculating the output $H$ of hidden layer by

$$
\begin{aligned}
H_{j} & =f\left(\sum_{i=1}^{n} w_{i j}-a_{j}\right) \quad j=1,2, \ldots, 1, \\
f(x) & =\frac{1}{1+e^{-x}}
\end{aligned}
$$

where $i$ represented the $i_{\text {th }}$ node of the input layer and $j$ was the $j_{\text {th }}$ node of the hidden layer, while $n$ expressed the node number of the input layer. $f(x)$ was the hidden layer excitation function.

Then, using the output $H$ of hidden layer the output of output layer $O$ was calculated in accordance with

$$
O_{k}=\sum_{j=1}^{1} H_{j} w_{j k}-b_{k} \quad k=1,2, \ldots, m,
$$

where $m$ expressed the node number of output layer, $k$ represented the $k_{\text {th }}$ node of the output layer, and $l$ was the hidden layer number.

Afterwards, the error $e$ of output layer should be calculated in accordance with

$$
e_{k}=y_{k}-O_{k} \quad k=1,2, \ldots, m,
$$

where $y_{k}$ was the desired output of output layer.

Then, the weights and thresholds of BPNN were updated in accordance with

$$
\begin{aligned}
& w_{i j}=w_{i j}+\eta H_{j}\left(1-H_{j}\right) x_{i} \sum_{k=1}^{m} w_{j k} e_{k} \\
& \quad i=1,2, \ldots, n ; j=1,2, \ldots, l ; \\
& w_{j k}=w_{j k}+\eta H_{j} e_{k} \quad j=1,2, \ldots, l ; k=1,2, \ldots, m, \\
& a_{j}=a_{j}+\eta H_{j}\left(1-H_{j}\right) x_{i} \sum_{k=1}^{m} w_{j k} e_{k} \quad j=1,2, \ldots, 1, \\
& b_{k}=b_{k}+e_{k} \quad k=1,2, \ldots, m,
\end{aligned}
$$

where $\eta$ represents the learning rate of BPNN.

Finally, iterative calculation should be made starting from the calculation of the output $H$ of hidden layer until meeting the required the end of iterative computation conditions. Once the iterative calculation had been completed, we can use the BPNN making classification for test samples. And, in this work, the node number of input layer, hidden layer, and output layer was 3, 4, and 3, respectively.

\section{Results and Discussion}

3.1. Characterization of $3 D$ Graphene Foam. The $3 \mathrm{D}$ graphene foam morphology was characterized by SEM, and the results were shown in Figures 5(a)-5(c) [27]. The graphene foam showed preeminent 3D interconnected networks and macroporous structure with pore diameters ranging from $\sim 200$ to $300 \mu \mathrm{m}$. This could offer a much larger specific surface area when compared to 2D graphene films. Additionally, the scaffold of thin graphene included many ripples and wrinkles on its surface on account of CVD growth on nickel substrate. This further increased the specific surface area of graphene.

The Raman spectrum of graphene foam was exhibited in Figure 5(d) [27]. The Raman profile displayed two protruding characteristic peaks at $1586.9 \mathrm{~cm}^{-1}$ and $2724.5 \mathrm{~cm}^{-1}$, corresponding to the $\mathrm{G}$ and $2 \mathrm{D}$ band of graphene, respectively. The intensity ratio between $\mathrm{G}$ and $2 \mathrm{D}$ band indicated that graphene foam was made of few layers of graphene. 


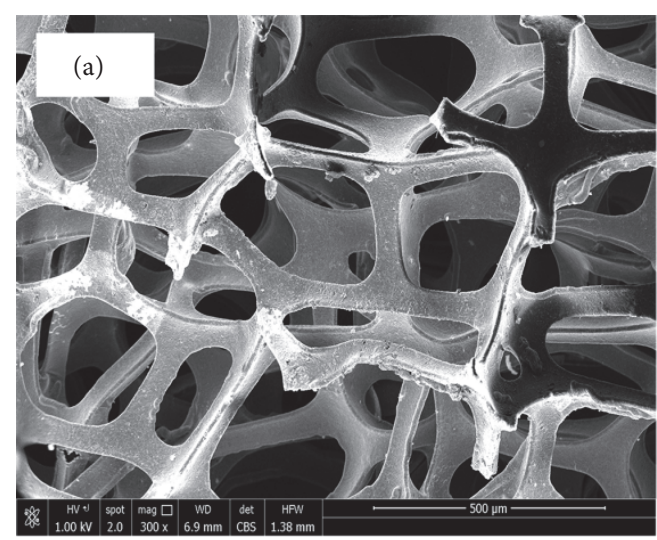

(a)

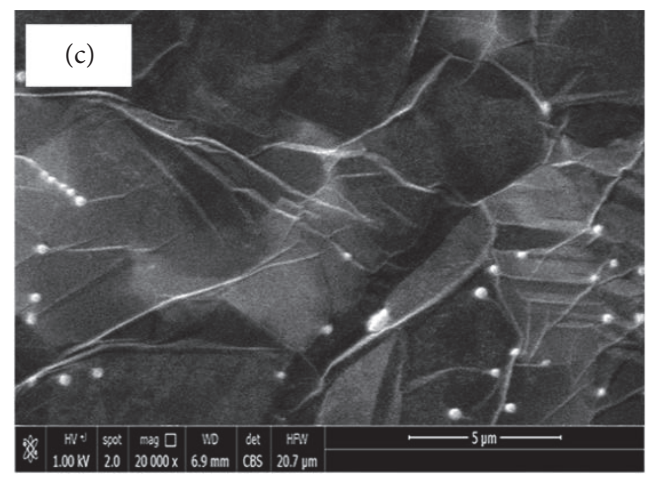

(c)

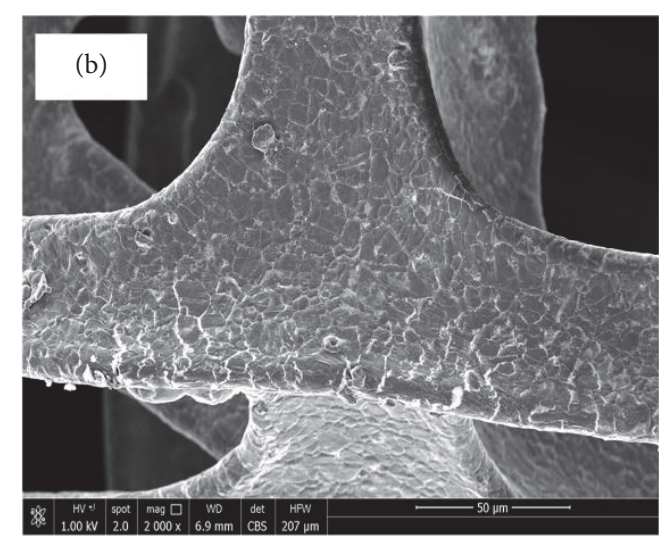

(b)

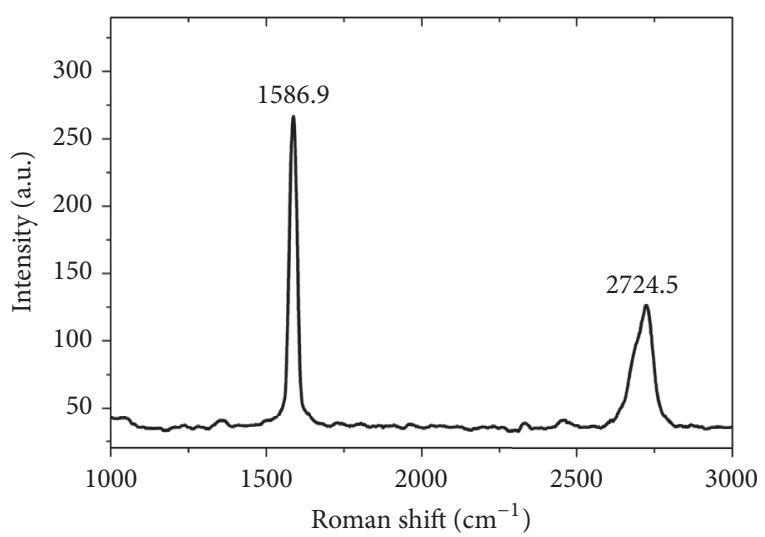

(d)

FIGURE 5: Characterization of graphene foam: $(\mathrm{a}-\mathrm{c})$ represent the morphology of foam using SEM and (d) depicts Raman spectra of 3D graphene foam.

3.2. Time Domain Resistance Measurement. The interaction of graphene foam with acetone, chloroform, and ether, was studied by recording the GFCSs real-time response using the homemade ERTDS real-time measurement of the fluctuant resistance as shown in Figure 6. A total of 135 curves were included in Figure 6 [27], while each kind of molecule included 45. Although various molecules produced different shape of response curves on the interface of graphene, all curves shared a common characteristic trend which showed first an ascending process followed by descending profile to finally return to stable state. The ascending section may indicate adsorption of the molecule on the interface of graphene foam, and the descending process may suggest the reserved process (desorption) due to volatile features of the molecules. The stable state may indicate that the molecules were completely pulled away from the graphene foam.

3.3. The Result of Data after PCA. Using the common characteristics, $t_{s}, t_{d}, t, A_{s}, A_{d}, A, R_{a s}, R_{a d}, V_{s}$, and $V_{d}$, ten characteristics of each curve were extracted. And for further decreasing the complexity of data and improving the efficiency of data processing, these ten characteristics were reduced by the method of PCA. And in this work ten characteristics of each curve were reduced to three characteristics. And the scatter plot of the three-dimensional coordinates which consisted of three characteristics was shown in Figure 7. And from Figure 7 we can see various molecules located in diverse area of three-dimensional coordinates.

3.4. Classification Accuracy. The GFCS adopting 135 sets of known data was used to build a complete topology structure of BPNN through BPNNT. The unseen samples were then measured and the complete topology structure of BPNN was used to predict the species, and the classification accuracy was shown in Figure 8. It can be seen that the classification accuracy of various molecules reached more than 97\% (acetone 97.82\%, chloroform 99.94\%, and ether 97.55\%).

Furthermore, the misjudgment situation of each molecule was investigated and the data were listed in Table 1. Using the BPNNC for acetone, the recorded probability of chloroform was $1.39 \%$ and that of ether was $0.78 \%$. For BPNNC as ether, the estimated probability for acetone was $0.05 \%$ and that of chloroform was $2.44 \%$. For BPNNC as chloroform, the recorded probability was only measured for ether $(0.06 \%)$. 


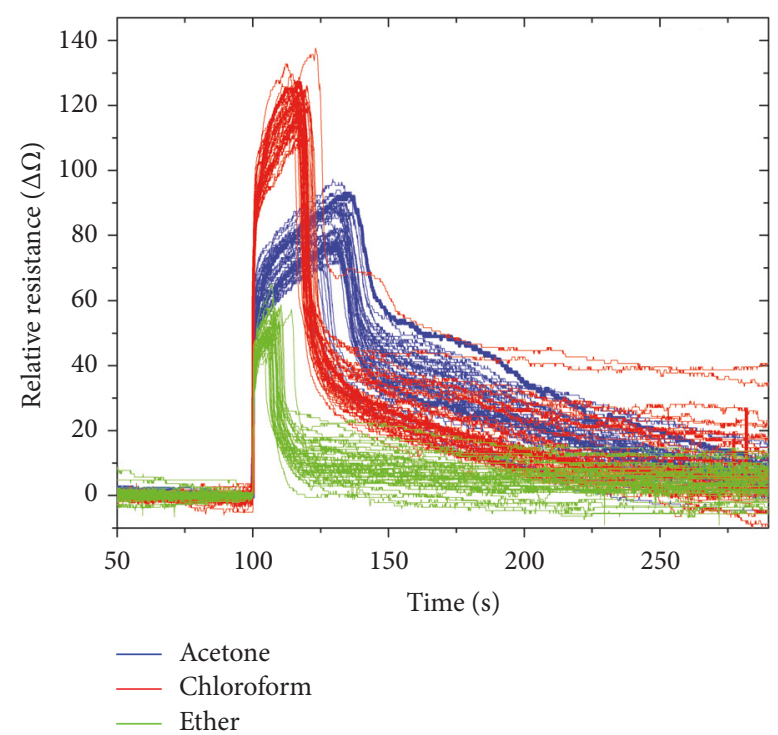

FIGURE 6: Time domain resistance curve of GFCS obtained with different chemical molecules: acetone, chloroform, and ether.

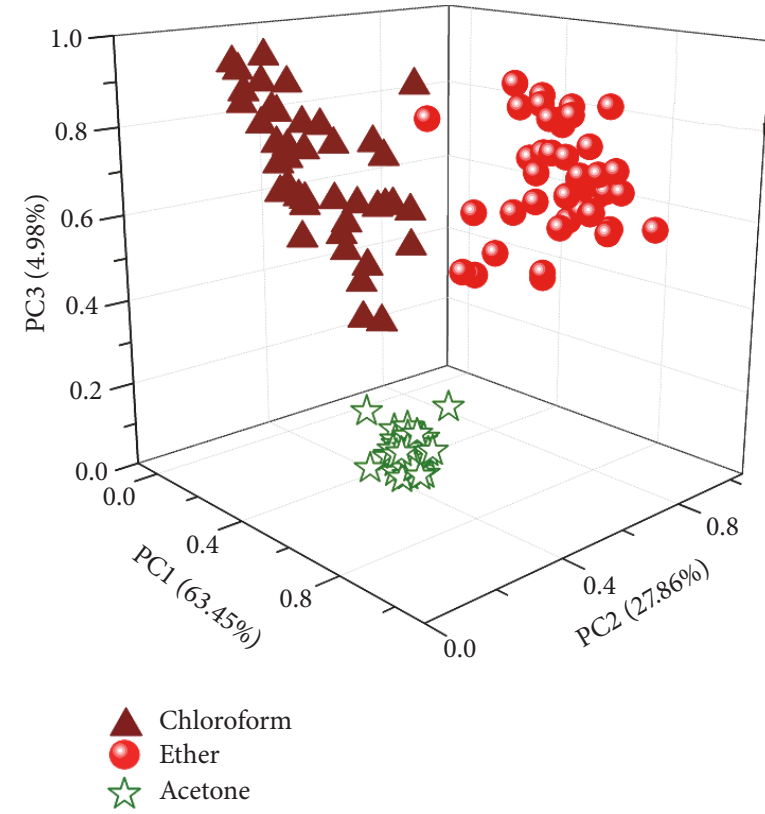

FIGURE 7: The scatter plot of diverse molecules response curves after DPP and PCA.

\section{Conclusions}

Using various time domain resistance curves produced by different molecules on the interface of graphene, an unmodified graphene foam chemical sensor system (GFCS) based on PCA and BPNN was constructed and tested for the identification of various chemical molecules, including acetone, chloroform, and ether. Unlike conventional chemical sensors requiring surface modification which could only identify specific single molecules, the GFCS could identify
TABLE 1: Accuracy and misjudgment situation of predicted results of each chemical molecule.

\begin{tabular}{lccc}
\hline Predicted result & \multicolumn{2}{c}{ Accuracy and misjudgment of predicted } \\
& Acetone & $\begin{array}{l}\text { results (\%) } \\
\text { Chloroform }\end{array}$ & Ether \\
\hline Acetone & 97.82 & 1.39 & 0.78 \\
Chloroform & 0 & 99.94 & 0.06 \\
Ether & 0.05 & 2.44 & 97.55 \\
\hline
\end{tabular}

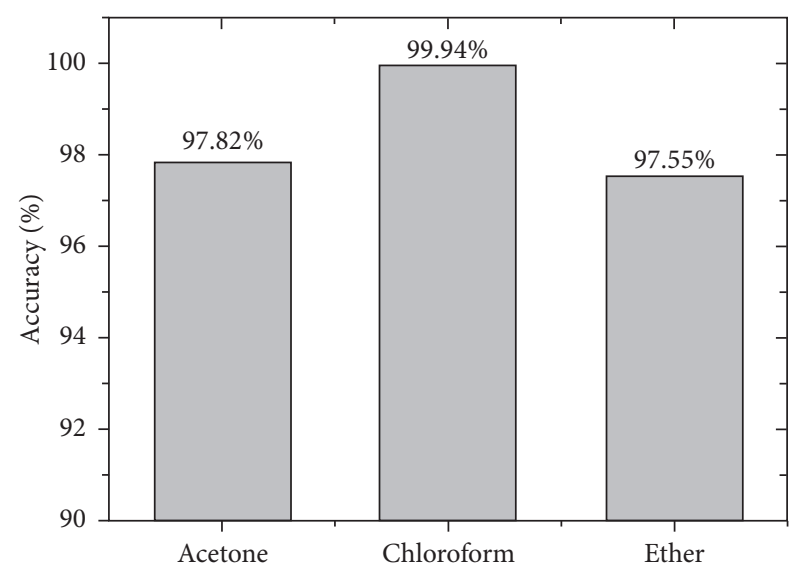

FIGURE 8: The classification accuracy of various molecules: acetone, chloroform, and ether.

various molecules without any modification through pattern recognition. The data showed that the predictive accuracy could reach above $97 \%$ for these chemical molecules, with excellent broad selectivity. Overall, these data might provide a new strategy for the design of graphene chemical sensors. Future work will focus on the development of new algorithms for targets discrimination in mixture compounds and optimization of the theoretical calculations.

\section{Disclosure}

Hongling Hua and Xiaohui Xie are co-first authors.

\section{Conflicts of Interest}

The authors declare that there are no conflicts of interest regarding the publication of this paper.

\section{Authors' Contributions}

Hongling Hua and Xiaohui Xie contributed equally to this work.

\section{Acknowledgments}

The research project was jointly supported by the National Natural Science Foundation of China (Grant nos. 61401258, 61205174, 61401259, and 11404193). 


\section{References}

[1] X. Li, J. Sha, S.-K. Lee et al., "Rivet Graphene," ACS Nano, vol. 10, no. 8, pp. 7307-7313, 2016.

[2] S. V. Morozov, K. S. Novoselov, and M. I. Katsnelson, "Giant intrinsic carrier mobilities in graphene and its bilayer," Physical Review Letters, vol. 100, no. 1, Article ID 016602, 2008.

[3] M. H. Kang, L. O. Prieto López, B. Chen et al., "Mechanical Robustness of Graphene on Flexible Transparent Substrates," ACS Applied Materials \& Interfaces, vol. 8, no. 34, pp. 2250622515, 2016

[4] K. S. Novoselov, A. K. Geim, S. V. Morozov et al., "Electric field in atomically thin carbon films," Science, vol. 306, no. 5696, pp. 666-669, 2004.

[5] F. Yavari and N. Koratkar, "Graphene-based chemical sensors," The Journal of Physical Chemistry Letters, vol. 3, no. 13, pp. 17461753, 2012.

[6] R. K. Paul, S. Badhulika, N. M. Saucedo, and A. Mulchandani, "Graphene nanomesh as highly sensitive chemiresistor gas sensor," Analytical Chemistry, vol. 84, no. 19, pp. 8171-8178, 2012.

[7] S. J. Park, O. S. Kwon, S. H. Lee, H. S. Song, T. H. Park, and J. Jang, "Ultrasensitive flexible graphene based field-effect transistor (FET)-type bioelectronic nose," Nano Letters, vol. 12, no. 10, pp. 5082-5090, 2012.

[8] B. Cai, S. Wang, L. Huang, Y. Ning, Z. Zhang, and G.-J. Zhang, "Ultrasensitive label-free detection of PNA-DNA hybridization by reduced graphene oxide field-effect transistor biosensor," ACS Nano, vol. 8, no. 3, pp. 2632-2638, 2014.

[9] N. Tammanoon, A. Wisitsoraat, C. Sriprachuabwong et al., "Ultrasensitive NO2 Sensor Based on Ohmic MetalSemiconductor Interfaces of Electrolytically Exfoliated Graphene/Flame-Spray-Made SnO2 Nanoparticles Composite Operating at Low Temperatures," ACS Applied Materials \& Interfaces, vol. 7, no. 43, pp. 24338-24352, 2015.

[10] Y. V. Stebunov, O. A. Aftenieva, A. V. Arsenin, and V. S. Volkov, "Highly Sensitive and Selective Sensor Chips with GrapheneOxide Linking Layer," ACS Applied Materials \& Interfaces, vol. 7, no. 39, pp. 21727-21734, 2015.

[11] X. Zhang, S. Xu, S. Jiang et al., "Growth graphene on silvercopper nanoparticles by chemical vapor deposition for highperformance surface-enhanced Raman scattering," Applied Surface Science, vol. 353, pp. 63-70, 2015.

[12] W. Yue, S. Jiang, S. Xu, Y. Ma, and C. Bai, "Fabrication of graphene FETs combined with fluorescence and its Double Read-Out System," Sensors and Actuators B: Chemical, vol. 214, Article ID 18216, pp. 204-210, 2015.

[13] W. Yue, S. Jiang, S. Xu, and C. Bai, "Fabrication of integrated field-effect transistors and detecting system based on CVD grown graphene," Sensors and Actuators B: Chemical, vol. 195, pp. 467-472, 2014.

[14] S. Xu, S. Jiang, J. Wang, J. Wei, W. Yue, and Y. Ma, "Graphene isolated Au nanoparticle arrays with high reproducibility for highperformance surface-enhanced Raman scattering," Sensors and Actuators B: Chemical, 2015.

[15] X. Dong, Y. Shi, W. Huang, P. Chen, and L.-J. Li, "Electrical detection of DNA hybridization with single-base specificity using transistors based on CVD-grown graphene sheets," Advanced Materials, vol. 22, no. 14, pp. 1649-1653, 2010.

[16] B. Jin, P. Wang, H. Mao et al., "Multi-nanomaterial electrochemical biosensor based on label-free graphene for detecting cancer biomarkers," Biosensors and Bioelectronics, vol. 55, pp. 464-469, 2014.
[17] Y. H. Kwak, D. S. Choi, Y. N. Kim et al., "Flexible glucose sensor using CVD-grown graphene-based field effect transistor," Biosensors and Bioelectronics, vol. 37, no. 1, pp. 82-87, 2012.

[18] S. Gupta Chatterjee, S. Chatterjee, A. K. Ray, and A. K. Chakraborty, "Graphene-metal oxide nanohybrids for toxic gas sensor: A review," Sensors and Actuators B: Chemical, vol. 221, pp. 1170-1181, 2015.

[19] S. Rumyantsev, G. Liu, M. S. Shur, R. A. Potyrailo, and A. A. Balandin, "Selective gas sensing with a single pristine graphene transistor," Nano Letters, vol. 12, no. 5, pp. 2294-2298, 2012.

[20] E. C. Nallon, V. P. Schnee, C. Bright, M. P. Polcha, and Q. $\mathrm{Li}$, "Chemical Discrimination with an Unmodified Graphene Chemical Sensor," ACS Sensors, vol. 1, no. 1, pp. 26-31, 2016.

[21] V. Dobrokhotov, A. Larin, and D. Sowell, "Vapor trace recognition using a single nonspecific chemiresistor., Sensors, vol. 13, no. 7, pp. 9016-9028, 2013.

[22] Z. Xie, X. Zuo, G.-P. Zhang, Z.-L. Li, and C.-K. Wang, "Detecting CO, NO and NO2 gases by Boron-doped graphene nanoribbon molecular devices," Chemical Physics Letters, vol. 657, pp. 18-25, 2016.

[23] I. Choudhuri, N. Patra, A. Mahata, R. Ahuja, and B. Pathak, "B-N@Graphene: Highly Sensitive and Selective Gas Sensor," The Journal of Physical Chemistry C, vol. 119, no. 44, pp. 2482724836, 2015.

[24] H. Liu, Z. Chen, S. Dai, and D.-E. Jiang, "Selectivity trend of gas separation through nanoporous graphene," Journal of Solid State Chemistry, vol. 224, pp. 2-6, 2015.

[25] Y. Ma, M. Zhao, B. Cai, W. Wang, Z. Ye, and J. Huang, “3D graphene foams decorated by $\mathrm{CuO}$ nanoflowers for ultrasensitive ascorbic acid detection," Biosensors and Bioelectronics, vol. 59, pp. 384-388, 2014.

[26] L. Wang, Y. Zhang, J. Yu et al., "A green and simple strategy to prepare graphene foam-like three-dimensional porous carbon/Ni nanoparticles for glucose sensing," Sensors and Actuators B: Chemical, vol. 239, pp. 172-179, 2017.

[27] W. Yue, H. Hua, Y. Tian et al., "An unmodified graphene foam chemical sensor based on SVM for discrimination of chemical molecules with broad selectivity," RSC Advances, vol. 7, no. 69, pp. 43560-43566, 2017.

[28] S. Aida, T. Matsuno, T. Hasegawa, and K. Tsuji, "Application of principal component analysis for improvement of X-ray fluorescence images obtained by polycapillary-based microXRF technique," Nuclear Instruments and Methods in Physics Research Section B: Beam Interactions with Materials and Atoms, vol. 402, pp. 267-273, 2017.

[29] M. Khodasevich, Y. Varaksa, G. Sinitsyn, V. Aseev, M. Demesh, and A. Yasukevich, "Determining the Stark structure of Yb3+ energy levels in $\mathrm{Y} 3 \mathrm{Al} 5 \mathrm{O} 12$ and $\mathrm{CaF} 2$ using principal component analysis of temperature dependences of fluorescence spectra," Journal of Luminescence, vol. 187, pp. 295-297, 2017.

[30] J. Gu, G. Yin, P. Huang, J. Guo, and L. Chen, "An improved back propagation neural network prediction model for subsurface drip irrigation system," Computers and Electrical Engineering, vol. 60 , pp. $58-65,2017$.

[31] Z. Xu, J. Liu, X. Chen, Y. Wang, and Z. Zhao, "Continuous blood pressure estimation based on multiple parameters from eletrocardiogram and photoplethysmogram by Back-propagation neural network," Computers in Industry, vol. 89, pp. 50-59, 2017. 
[32] Z.-L. Zhang, X.-G. Luo, S. García, and F. Herrera, "CostSensitive back-propagation neural networks with binarization techniques in addressing multi-class problems and noncompetent classifiers," Applied Soft Computing, vol. 56, pp. 357367, 2017.

[33] Z. Chen, W. Ren, L. Gao, B. Liu, S. Pei, and H.-M. Cheng, "Three-dimensional flexible and conductive interconnected graphene networks grown by chemical vapour deposition," Nature Materials, vol. 10, no. 6, pp. 424-428, 2011.

[34] P. Trinsoutrot, H. Vergnes, and B. Caussat, "Three dimensional graphene synthesis on nickel foam by chemical vapor deposition from ethylene," Materials Science and Engineering: B Advanced Functional Solid-State Materials, vol. 179, no. 1, pp. 12-16, 2014.

[35] W. Jiang, H. Xin, and W. Li, "Microcellular 3D graphene foam via chemical vapor deposition of electroless plated nickel foam templates," Materials Letters, vol. 162, pp. 105-109, 2016. 

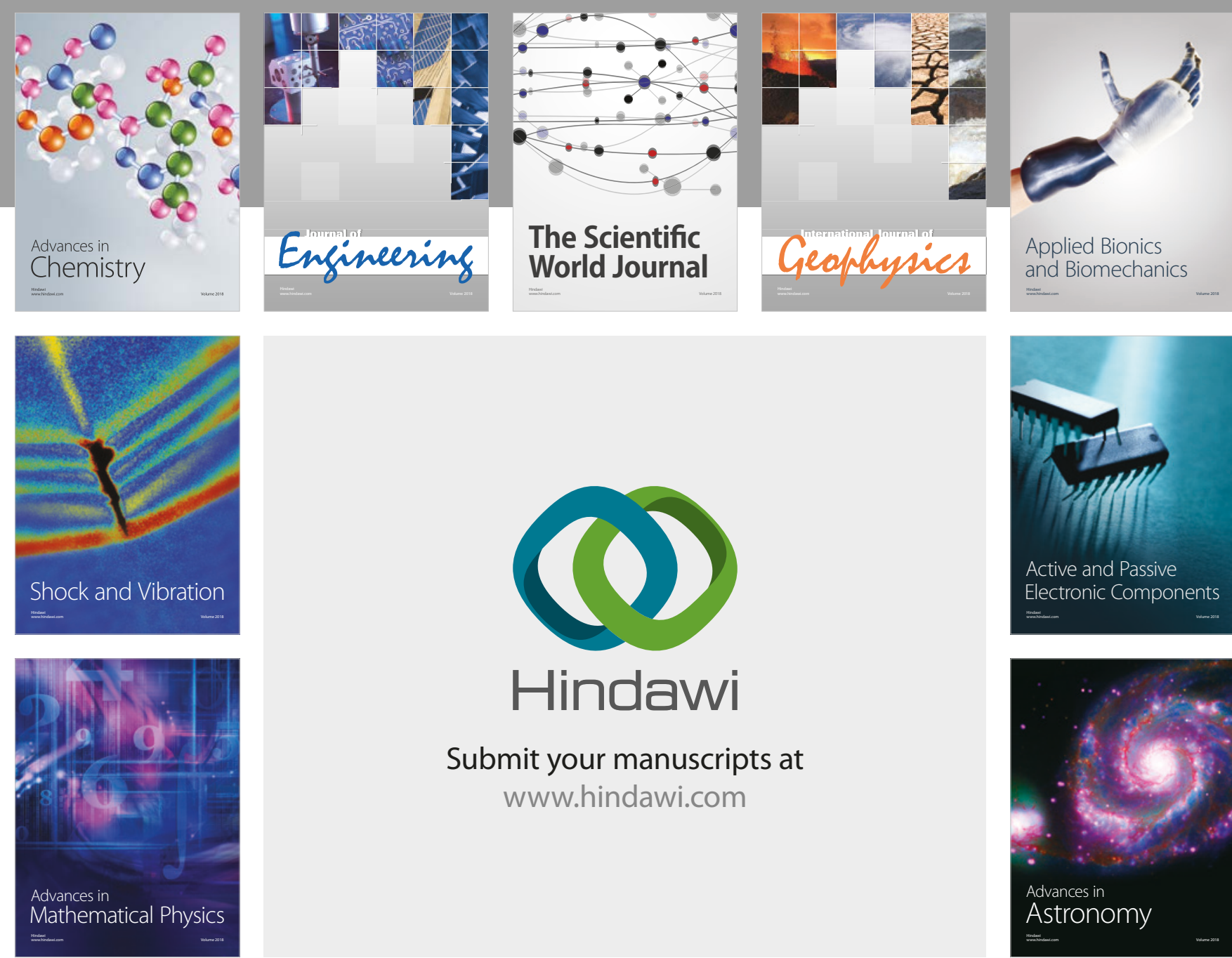

Submit your manuscripts at

www.hindawi.com

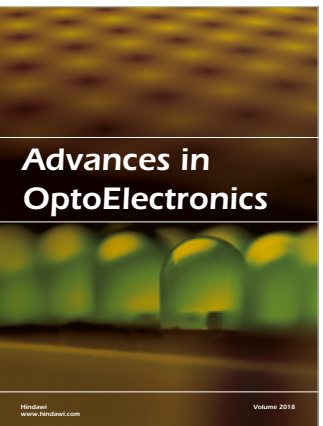

\section{Rotcting Machinery}
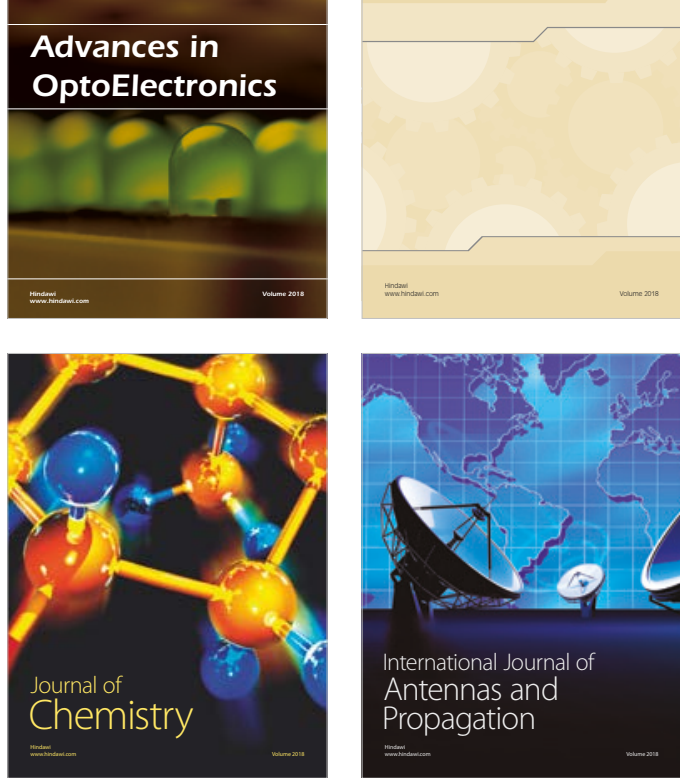

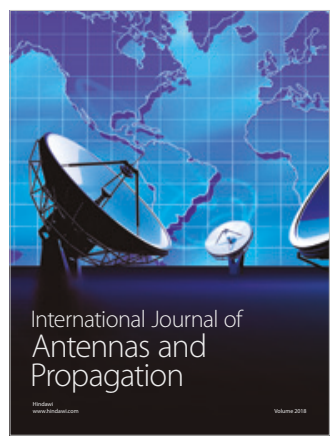

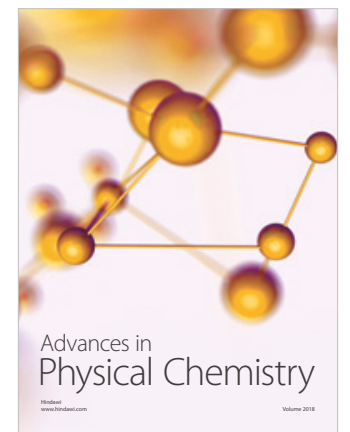

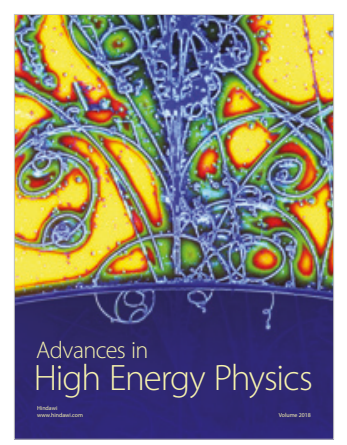

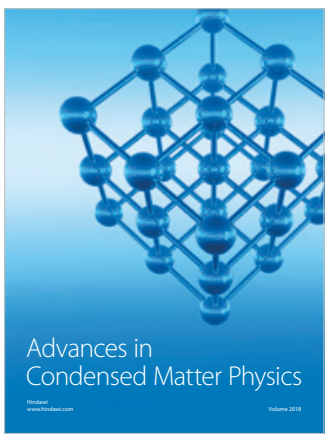

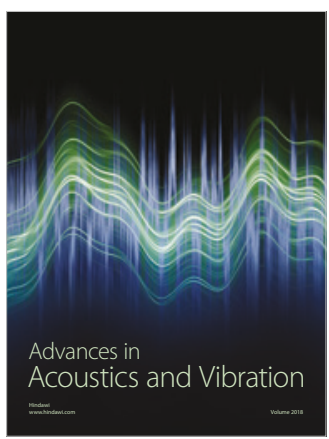

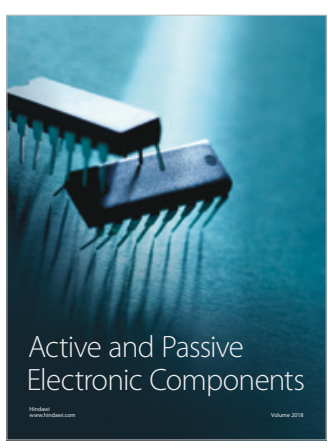
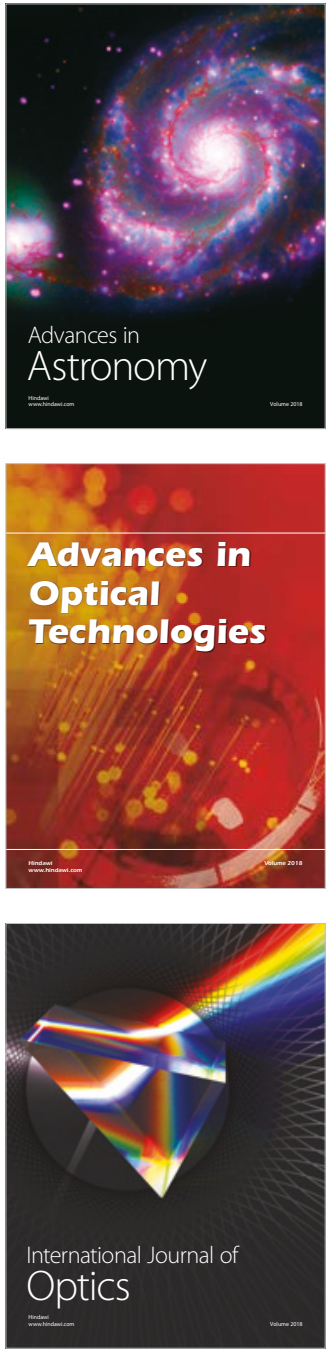